\title{
The relationship between the perception of sweet and salty tastes and the addition of potato starch
}

\author{
Yoko Oкамото $^{1)}$, Saori Hisano ${ }^{1)}$ and Keiko YoshidA ${ }^{2)}$ \\ ${ }^{1)}$ Suzugamine Women's College, 4-6-18, Inokuchi, Nishi-ku, Hiroshima 733-8623, Japan \\ ${ }^{2)}$ Tsukuba International Junior College, 6-7-26, Manabe, Tsuchiura, 300-0051, Japan
}

The taste intensity (taste efficacy) of either gel samples alone or sol samples alone has been examined in previous studies. In the present study, by contrast, the concentration of potato starch in solutions of taste compounds was varied from 0.16 to $20.00 \%$, thereby making it possible to examine the intensity of sweetness and saltiness in both gel and sol samples under identical conditions. On the transition of sweet and salty sol samples to gel samples, decreases in taste intensity were found in this study. In addition, significant differences were found in taste intensity on comparison of a large number of pairs of gel and sol samples. Therefore, we may conclude that there are significant differences in taste intensity between gel and sol samples. The results of the rupture stress measurements of the starch-added sucrose samples showed that rupture stress rose markedly on the transition from sol to gel. Taking the results of this study together, we conclude that when a taste sample shifts from a sol to a gel and the viscosity and hardness of the sample increases, then the human taste sensation is weakened concomitantly.

(Received 10 January 2007, Accepted 28 May 2007)

Keywords: sweet taste, salty taste, sensory evaluation, potato starch, rupture stress

\section{Introduction}

It is well known that taste sensitivity to food decreases with increases in concentration, viscosity and, in the case of gels, hardness. In previous studies of changes in taste sensitivity due to variations in the physical and mechanical properties of foods, the tastes of gustatory compounds have been mostly compared as either exclusively gel samples or exclusively solution (sol) samples (Moritaka and Naito 2002 ; Moritaka and Shimada 2005 ; Wilson and Brown 1997 ; Yamaguchi 1980 ; Yamamoto and Nakabayashi 1999).

In this study, potato starch was used to give viscosity to solutions. By adding starch, sweet or salty samples were transformed from sol to gel, and the tastes of sweet and salty compounds in the sol samples were compared with those in the gel samples under identical conditions.
To study how human taste sensitivity to representative sweet and salty compounds is altered by increases in the concentration of added starch, the following issues were examined. Because generally the border between sol and gel cannot be determined exactly, it was first evaluated how the rupture properties of a solution of sucrose, as a representative sweet substance, changed with increases in the concentration of added starch. On the basis of these measurements, a criterion differentiating gel from sol was defined for this study. How the 'starch-added sweet or salty samples' differed from the 'solutions of sweet or salty compounds' (i. e. the standard solutions) in terms of the intensity of sweetness or saltiness was subsequently determined for ten kinds of sweet substance and two salty substances using the sensory evaluation method. In addition, the relationship between the sensory evaluation and the rup- 
Table 1. Sweet or salty samples

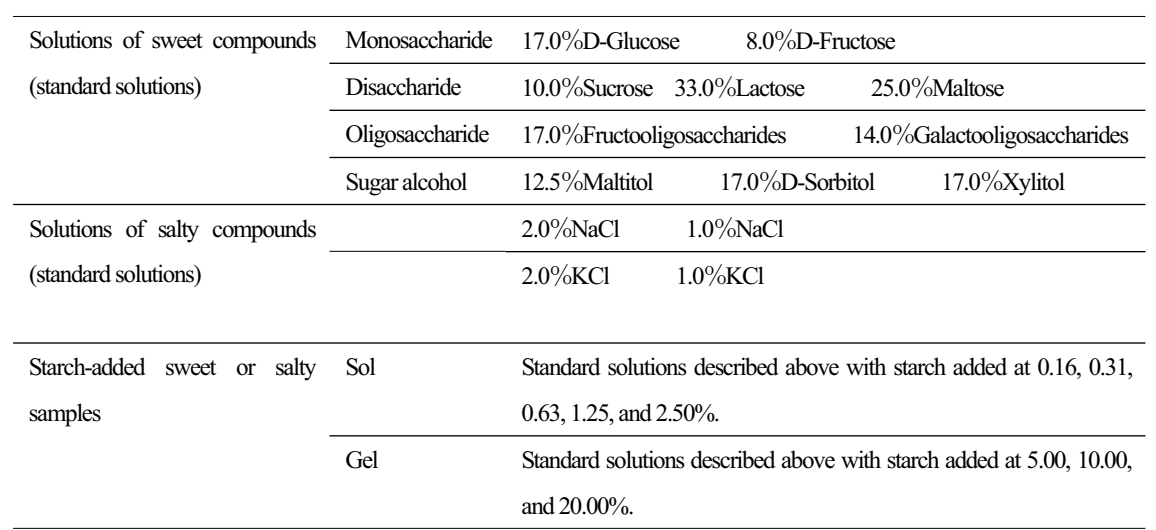

Concentrations of sweet and salty compounds and starch are expressed as percentage by weight $(\%, \mathrm{~W} / \mathrm{V})$.

Fructo-oligosaccharide, and galacto-oligosaccharide were purchased from Meiji-Seika Inc., and Calpis Inc., respectively. Other compounds were purchased from Sigma-Aldrich Japan Inc.

ture stress was clarified for the 'starch-added sucrose solutions'.

\section{Materials and Methods}

\section{Samples}

The sweet and salty samples used in the experiments are shown in Table 1. For the sweet samples, solutions of sweet compounds with a sweetness almost equal to that of $10.0 \%$ sucrose were prepared taking the degree of sweetness into consideration (Aoki 2002). Sucrose at this concentration in standard soft drinks gives us a sweet taste sensation (Yamazaki et al. 2003). For the salty samples, $\mathrm{NaCl}$ and $\mathrm{KCl}$ solutions were prepared at 1.0 and $2.0 \%$. Salty compounds at these concentrations in savory foods give us a salty taste sensation (Yamazaki et al. 2003).

To prepare the 'starch-added samples', starch was dissolved in purified water, and then one of taste substances was added. The solution thus prepared was heated to $80^{\circ} \mathrm{C}$ to gelatinate the starch. The physical and mechanical properties of gelatinated starch can vary markedly depending both on stirring conditions during the heating process and on the prior history of heating (Fuwa et al. 2004). Consequently, the solution was heated carefully so that its temperature rose to $80^{\circ} \mathrm{C}$ in exactly 10 minutes.
The solution was weighed before and after heating, and water equivalent to the amount evaporated during heating was added. The 'starch-added samples', heated as described above, were cooled to room temperature and kept in a thermostat water bath at $25^{\circ} \mathrm{C}$ for 5 minutes before use. The interval between completion of the gelatination of starch-added samples and measurement of the rupture property or sensory evaluation was less than 60 minutes. Concentrations of sweet and salty compounds and starch are expressed as a percentage by weight (\%, $\mathrm{W} / \mathrm{V})$.

On the basis of measurements of the rupture stress of the 'starch-added sucrose samples' (Fig. 2) and their properties, samples containing starch at concentrations of $0.16,0.31,0.63,1.25$ and $2.50 \%$ were defined as sols, and those containing starch at concentrations of $5.00,10.00$, and $20.00 \%$ were defined as gels at the temperature at which the experiments were conducted. In this study, the rupture stress of samples other than the 'starch-added sucrose samples' was not measured. In accordance with the 'starch-added sucrose samples', however, samples of other sweet and salty compounds containing starch at concentrations of $0.16,0.31,0.63,1.25$, and $2.50 \%$ were also defined as sols, and those containing starch at concentrations of $5.00,10.00$, and $20.00 \%$ 
The relationship between the perception of sweet and salty tastes and the addition of potato starch

were defined as gels (Table 1$)$.

2. Measurement of the rupture stress

The rupture stress of each gel in a stainless steel dish was measured by a rheometer (Yamaden Co. Ltd., RE2-3305B, Tokyo, Japan). Samples were placed in a stainless steel dish with an inner diameter of $40 \mathrm{~mm}$ and a depth of $15 \mathrm{~mm}$. The plunger diameter was $20 \mathrm{~mm}$, the sample stage speed was 10 $\mathrm{mm} / \mathrm{s}$ and the temperature was $25^{\circ} \mathrm{C}$. Samples were measured at a compression strain ratio of $60 \%$. The load cell was set at 2 to $20 \mathrm{~N}$. Measurements were made after the samples had been kept at $25^{\circ} \mathrm{C}$ for 5 minutes in a water bath, and were repeated six times for each sample.

\section{Sensory evaluation}

We examined the intensity of sweetness and saltiness experienced by each panelist by using the scoring system of the sensory evaluation method (Yamano et al. 2003). A 7-point scale, from-3 to +3 with two limits, was used (Yamano et al. 2003).

The panel consisted of 20-26 healthy female junior college students aged between 18 and 20 years. The sensory evaluation was performed between 2 and 4 $\mathrm{PM}$ and was more than 1 hour after lunch.

The panel tasted the 'standard sweet solution', followed by the 'starch-added sweet sample', and then evaluated the relative intensities of their sweetness according to the following scale. When the 'starchadded sweet sample' was very sweet as compared with the 'standard solution', a score of +3 was given. When it was moderately sweet, a score of +2 was given. When it was slightly sweet, not different in sweetness, slightly less sweet, moderately less sweet, and markedly less sweet, scores of $+1,0,-1,-2$, -3 , respectively, were given in a similar manner. The sensory evaluation was made in the same way for the 'starch-added salty samples'.

When presented with a sample, the panel tasted it with the whole mucosal surface of the tongue and oral cavity. Before a sample was tasted, the oral cavity was rinsed with purified water to remove remnants of samples tasted previously. Samples were expectorated after tasting in order to avoid becoming replete. The sensory evaluation was made in an air-conditioned, quiet room separated from other rooms (a room for experiment preparation). This room had a water supply and a sink for rinsing the mouth. A 'starch-added sample' was placed in the mouth with a plastic cup with a $15-\mathrm{ml}$ volume if it was a sol, and with a plastic spoon if it was a gel. Gel samples were chewed once in the mouth.

\section{Data analysis}

Sensory evaluation scores are expressed as the mean (hereafter referred to as mean score) \pm SD. The score data were analyzed by one-way layout analysis of variance, and differences between groups were tested with Tukey's multiple comparison (Ishimura 2002). A p-value $<0.05$ was considered significant.

Rupture stress values are expressed as the mean $\pm \mathrm{SD}$. Correlation coefficients between the rupture stress values and the sensory evaluation scores were calculated.

SPSS 11.5J software for Windows (SPSS Japan Inc.) was used for statistical analysis.

\section{Results}

1. Sensory evaluation scores representing intensities of sweetness and saltiness

Intensities of sweetness and saltiness were compared between 'standard solutions' and 'starch-added taste samples' (Fig. 1).

As shown in Figure 1, the mean scores decreased as the concentration of starch increased in the "starch -added samples' of any of the sweet and salty compounds. In other words, both the sweet and salty taste sensations decreased in intensity concomitantly with the increase in starch concentration. In view of these changes in the mean score, it is considered that the intensity of taste was lower in the gel samples than in the sol samples. There were no marked differences in this trend among the sweet and salty compounds tested in this study.

2. Multiple comparison of sensory evaluation data 

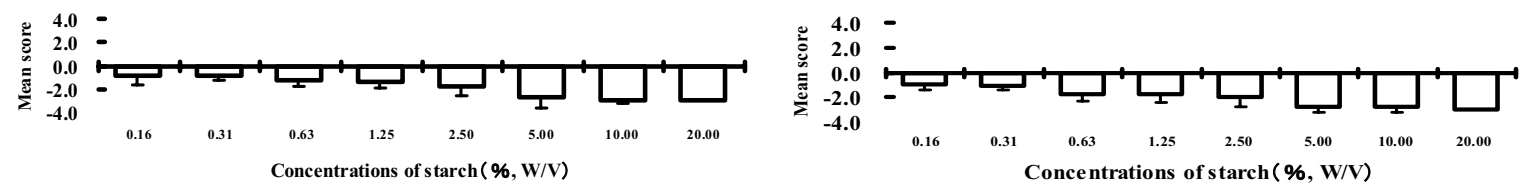

Starch-adde d sucrose

Starch-added lactose
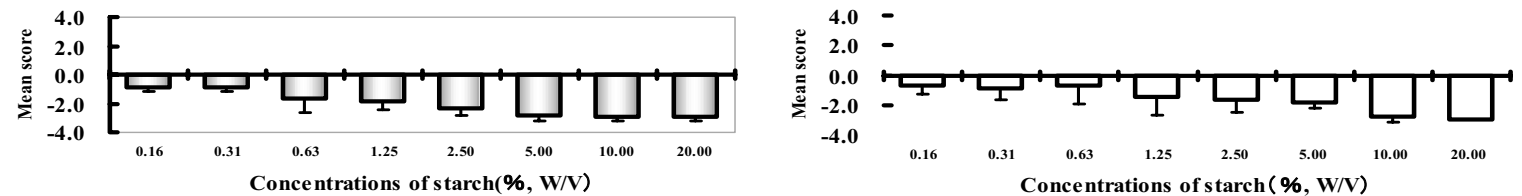

Starch-added maltos

Starch-added fructooligos accharides

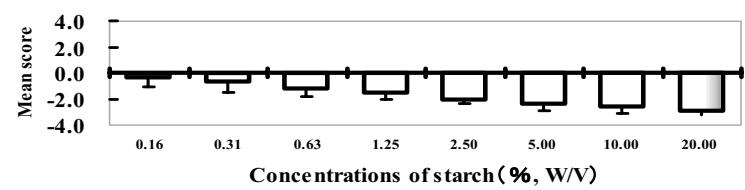

$4.0-$

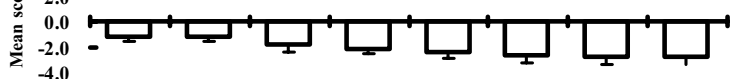

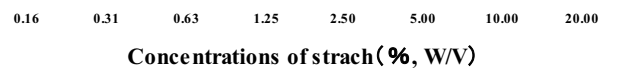

Starch-added Galactooligos accharides

Starch-added maltitol
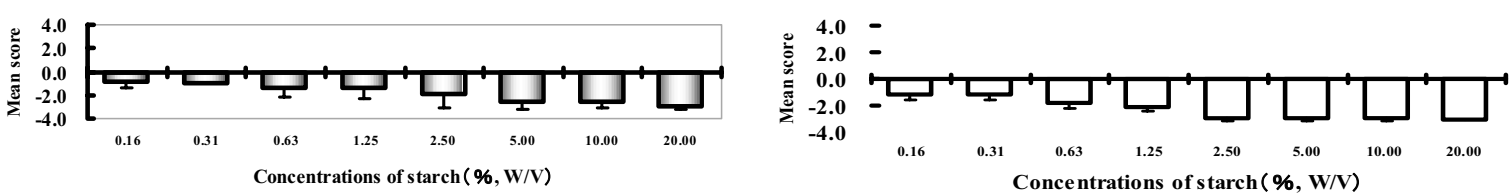

Starch-added D-sorbito

Starch-added xylito
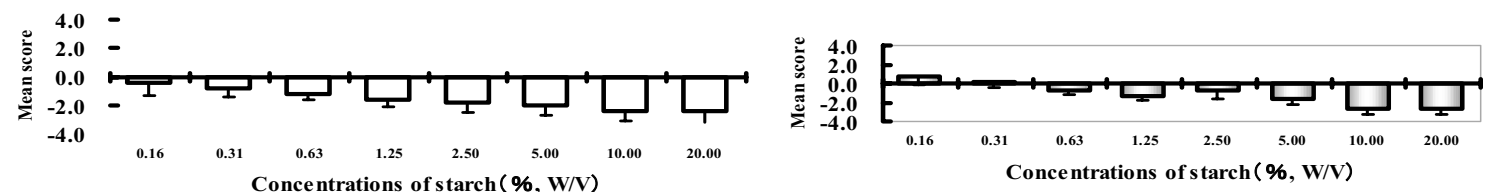
The relationship between the perception of sweet and salty tastes and the addition of potato starch

Starch-adde $2.0 \% \mathrm{NaCl}$

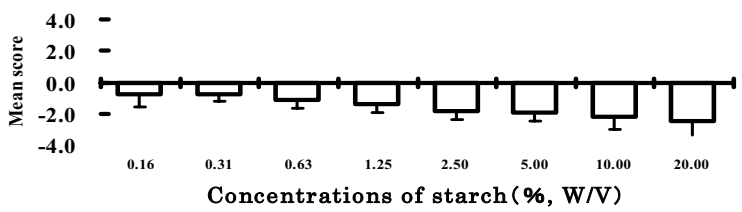

starch-adde d $1.0 \% \mathrm{NaCl}$

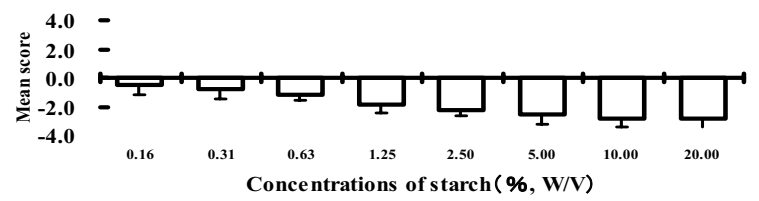

Starch-added $2.0 \% \mathrm{KCl}$

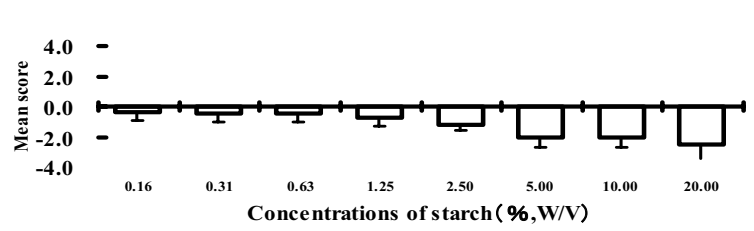

Starch-added $1.0 \% \mathrm{KCl}$

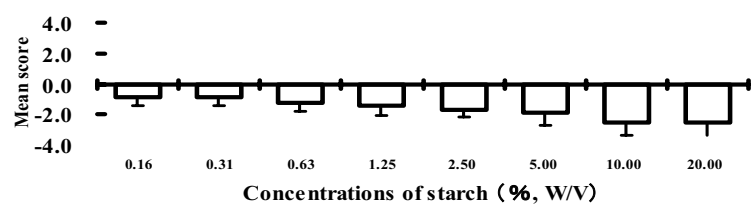

Figure 1. Sweetness and saltiness intensities of starch-added samples.

The sweetness and saltiness intensities of 'starch-added samples' were compared with those of standard solutions, and are shown as the mean score \pm SD. A smaller mean score of a 'starch-added sample' means that the taste intensity of that sample was lower.

Multiple comparison was carried out on the sensory evaluation data for each of ten series of sweet samples, where each series contained an identical kind of sweet compound at an identical concentration and starch at successively increasing concentrations. Similarly, multiple comparison was done for each of four series of salty samples, two of which contained $\mathrm{NaCl}$ at $1.0 \%$ and $2.0 \%$, and two of which contained $\mathrm{KCl}$ at $1.0 \%$ and $2.0 \%$.

The results of the multiple comparison of the sensory evaluation data obtained for starch-added Dglucose samples, and those for starch-added $2.0 \%$ $\mathrm{NaCl}$ samples are shown in Tables 2 and 3, respectively. Similar results were obtained for the samples not shown in these tables. When the mean score of the taste intensity was compared between a sol sample and another sol sample, or between a gel sample and another gel sample, no significant differences were found in most pairs of samples (132 of 182 pairs). When the mean score was similarly compared between a gel sample and a sol sample, by contrast, there were more pairs with significant differences than without significant differences (167 of 210 pairs).
3. Relationship between the rupture stress and sensory evaluation score in starch-added sucrose samples

Figure 2 shows the rupture stress values and the mean evaluation scores of the starch-added sucrose samples. As the starch concentration increased, the rupture stress values increased, whereas the mean sensory evaluation score decreased. A negative correlation was found between the rupture stress and sweetness intensity (Pearson's correlation efficient, $r$ $=-0.598)$.

Thus, as ascertained by the sensory evaluation method and a rheometer, increases in the viscosity of sucrose samples (i. e. increases in the rupture stress) lead to a decrease in the intensity of sweetness.

The sweet taste sensation was found to be markedly low in the range of starch concentrations at which the rupture stress of samples was greatest. The sweetness intensity of gel samples was considered to be markedly lower than that of sol samples at $25^{\circ} \mathrm{C}$, the temperature at which the experiments were made. 


\section{日本官能評価学会誌 Vol. 11 No. 2}

Table 2. Results of multiple comparison of sensory evaluation data for pairs of starch-added D-glucose samples

\begin{tabular}{lcccccccc}
\hline & 0.16 & 0.31 & 0.63 & 1.25 & 2.50 & 5.00 & 10.00 & 20.00 \\
\hline 0.16 & & & & & & & & \\
0.31 & $\mathrm{~ns}$ & & & & & & & \\
0.63 & $\mathrm{~ns}$ & $\mathrm{~ns}$ & & & & & & \\
1.25 & $\mathrm{~ns}$ & $\mathrm{~ns}$ & $\mathrm{~ns}$ & & & & & \\
2.50 & $*$ & $*$ & $\mathrm{~ns}$ & $\mathrm{~ns}$ & & & & \\
5.00 & $*$ & $*$ & $*$ & $*$ & $*$ & & & \\
10.00 & $*$ & $*$ & $*$ & $*$ & $*$ & $\mathrm{~ns}$ & & \\
20.00 & $*$ & $*$ & $*$ & $*$ & $*$ & $\mathrm{~ns}$ & $\mathrm{~ns}$ & \\
\hline
\end{tabular}

Numbers in the uppermost row and the left column indicate concentrations of starch $(\%, \mathrm{~W} / \mathrm{V})$.

$*: \mathrm{p}<0.05$, ns : Not significant

Table 3. Results of multiple comparison of sensory evaluation data for pairs of starch-added $2.0 \%$ $\mathrm{NaCl}$ samples

\begin{tabular}{|c|c|c|c|c|c|c|c|c|}
\hline & 0.16 & 0.31 & 0.63 & 1.25 & 2.50 & 5.00 & 10.00 & 20.00 \\
\hline \multicolumn{9}{|l|}{0.16} \\
\hline 0.31 & ns & & & & & & & \\
\hline 0.63 & $\mathrm{~ns}$ & ns & & & & & & \\
\hline 1.25 & $\mathrm{~ns}$ & ns & ns & & & & & \\
\hline 2.50 & $*$ & $*$ & ns & $\mathrm{ns}$ & & & & \\
\hline 5.00 & $*$ & * & $*$ & $\mathrm{~ns}$ & ns & & & \\
\hline 10.00 & $*$ & $*$ & * & $*$ & ns & $\mathrm{ns}$ & & \\
\hline 20.00 & $*$ & $*$ & * & $*$ & ns & ns & ns & \\
\hline
\end{tabular}

Numbers in the uppermost row and the left column indicate concentrations of starch $(\%, \mathrm{~W} / \mathrm{V})$.

$*: \mathrm{p}<0.05$, ns : Not significant

\section{Discussion}

In a previous study, in which taste intensity was examined in solutions containing cornstarch at concentrations of 2-10\% (Yamamoto and Nakabayashi 1999), marked decreases in taste intensity were found as the viscosity of the solutions increased. Taste intensity was examined only in cornstarchadded sol samples in that study (Yamamoto and Nakabayashi 1999), whereas it was examined in sol samples containing potato starch at 0.16, 0.31, 0.63, 1.25 , and $2.50 \%$ in the present study. The results of the present study are essentially in accordance with those of the previous study using cornstarch-added sol samples.
Yamaguchi (1980) compared the taste intensity of sweet compounds in potato starch gel with that of compounds dissolved in water, and found a propensity for the taste intensity to decrease with stepwise increases in potato starch concentration from $10 \%$, to $20 \%$, and finally to $30 \%$. Whereas only gel samples were used in the study of Yamaguchi (1980), gel samples prepared with $5.00,10.00$, and $20.00 \%$ potato starch were used in the present study. The results of the present study are essentially in agreement with those of the study of Yamaguchi (1980).

Moritaka and Shimada (2005) studied the taste intensity of agar gels and gellan gum gels containing salty compounds. They showed that, irrespective of 
The relationship between the perception of sweet and salty tastes and the addition of potato starch

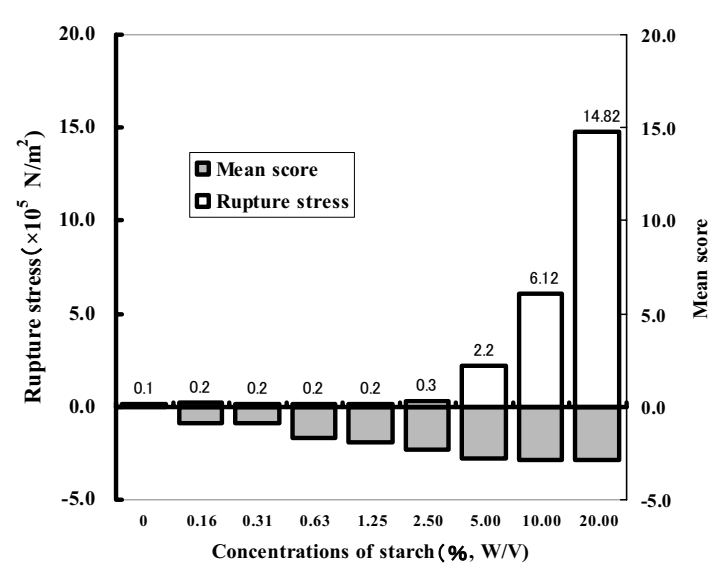

Figure 2. Relationship between the rupture stress and the sensory evaluation score of starch-added sucrose samples.

Each column shows a mean value. Numbers on the abscissa indicate concentrations of starch added to the sucrose solution. 'Zero' on the abscissa corresponds to a sucrose solution containing no starch.

whether agar or gellan gum was used, the salty taste intensity in soft gel samples was higher than that in firm gel samples. Similar results were obtained for salty gel samples in the present study.

As described above, the taste intensity (taste effcacy) of either gel samples alone or sol samples alone has been examined in previous studies (Moritaka and Naito 2002 ; Moritaka and Shimada 2005 ; Wilson and Brown 1997 ; Yamaguchi 1980 ; Yamamoto and Nakabayashi 1999). In the present study, by contrast, the concentration of potato starch in solutions of taste compounds was varied from 0.16 to $20.00 \%$, thereby making it possible to examine the intensity of sweetness and saltiness in both gel and sol samples under identical conditions. On the transition of sweet and salty sol samples to gel samples, decreases in taste intensity were found in this study. In addition, significant differences were found in taste intensity on comparison of a large number of pairs of gel and sol samples. Therefore, we may conclude that there are significant differences in taste intensity between gel and sol samples. In this study, ten kinds of sweet compound and two kinds of salty compound at two different concentrations were used. The fact that the data were obtained using not just one or two representative taste compounds but a variety of different compounds seems to make the results more robust.

The relationship between the rupture stress (gel firmness) and the sensory evaluation was clarified only for the 'starch-added sucrose samples', and not for other samples. Nevertheless, the relationship between the rupture stress and the sensory evaluation for the 'starch-added sucrose samples' is expected to hold true for starch-added samples of other taste compounds. This notion, however, must be confirmed in future studies.

The decrease in taste intensity that occurs as the concentration of starch added to solutions of taste compounds increases may be explained as follows (Yamano et al. 2003). Because a solution can easily spread in the mouth and gain access to many taste cells, a taste compound in a solution is probably able to induce a maximal sensation. Because solid or gel foods cannot be swallowed unless they are chewed, they are mixed with saliva and the taste compounds within them become diluted. As a consequence, the intensity of their sweetness or saltiness is lowered. In addition, it is less easy and takes longer for taste compounds in viscous and hard foods to gain access to taste cells as compared with those in liquid and soft foods. This may be a reason why viscous and hard foods have less taste than liquid and soft foods.

\section{References}

Aoki, T. (2002) Shin Shyokuhingaku Soron Kakuron (in Japanese), Asakurasyoten, Tokyo, pp.105.

Fuwa, E., Komaki, T., Hizukuri, S., and Kainuma, K. (2004) Denpunkagaku No Jiten (in Japanese), Asakurasyoten, Tokyo, pp.183-186.

Ishimura, S. (2002) SPSS Niyoru Bunsanbunseki To

Tajuhikaku No Tejun (in Japanese), Tokyosyoseki, Tokyo, pp.2-29.

Moritaka, H. and Naito, S. (2002) Agar and gelatin gel flavor release, Journal of Texture Studies, 33, 201-214.

Moritaka, H. and Shimada, A. (2005) Effects of potassium chloride and calcium chloride on mechani- 


\section{日本官能評価学会誌 Vol. 11 No. 2}

cal properties and salt intensities of agar gel and gellan gum gel (in Japanese), Japanese Journal of Sensory Evaluation, 9 (2), 108-115.

Wilson, C. E. and Brown. W. E. (1997) Influence of food matrix structure and oral breakdown during mastication on temporal perception of flavor, Journal of Sensory Studies, 12, 69-86.

Yamaguchi, S. (1980) The interaction of a texture and the taste (in Japanese), Syokuhin No Bussei, Syokuhinsizai-kenkyukai, 6, 143-152.
Yamamoto, Y. and Nakabayashi, M. (1999) Effects of viscosity on the intensity of salt or sweet taste in starch solutions (in Japanese), Journal of Cookery Science of Japan, 32 (4), 334-337.

Yamano, Y. (2003) Oishisa No Kagaku Jiten, Asakurasyoten, Tokyo, pp.19-22, 90-97.

Yamazaki, K., Shimada, K., Shibukawa, S., and Shimomura, M. (2003) Cookery and its Theory (in Japanese), Dobunsyoin, Tokyo, pp.522-523. 